\title{
Depapillated tongue with keratotic papules: What's your diagnosis?
}

\author{
Idoudi Safa', Soua Yosra', Bouattay Rachida', Ben Abdeljelil Nouha ${ }^{3}$
}

${ }^{1}$ Department of Dermatology, Fattouma Bourguiba University Hospital, Monastir-Tunisia, ${ }^{2}$ Department of Otorhinolaryngology, Fattouma Bourguiba University Hospital, Monastir-Tunisia, ${ }^{3}$ Department of Anapathology, Fattouma Bourguiba University Hospital, Monastir-Tunisia

Corresponding author: Dr. Idoudi Safa, E-mail: safaa.idoudi@gmail.com

\section{CASE REPORT}

A 23-year-old female patient consulted our department for burning sensation and painful tongue for the past 3 months. These burning sensations are aggravated on intake of hot and spicy foods. Patient reported no history of drug intake before. Examination revealed erythematous depapillated tongue on its dorsal surface surmounted by multiple white keratotic papules (Fig. 1).

Dermoscopic examination revealed white patch with thin reticular striae surrounded with erythematous area and curved vessels (Fig. 2).

The rest of the clinical examination didn't show any signs of associated buccal mucosa, ocular, cutaneous, or genital lesions.

\section{HISTOLPATHOLOGY}

Histopathological examination revealed irregular acanthosis, basal layer vacuolization and necrotic keratinocytes (Fig. 3A).

Note the presence at the dermoepidermal junction of a moderately dense interface dermatitis (Fig. 3B).

The rest of the dermis was without abnormalities.

The mycological examination revealed no mycelium.

\section{WHAT'S YOUR DIAGNOSIS?}

Answer: Oral lichen planus of the tongue.

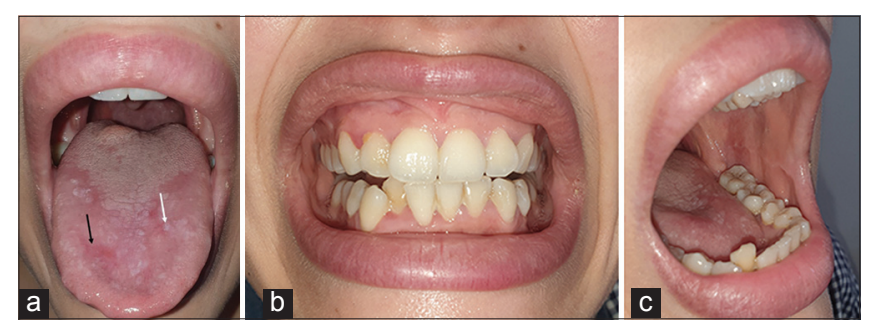

Figure 1: (a) Diffuse deflated patch with irregular margin on the tip of the tongue (black arrow) surrounded with multiple white keratotic papules on the dorsum of the tongue (white arrow). (b) No gum involvement. (c) No lesions of inside of the cheek.

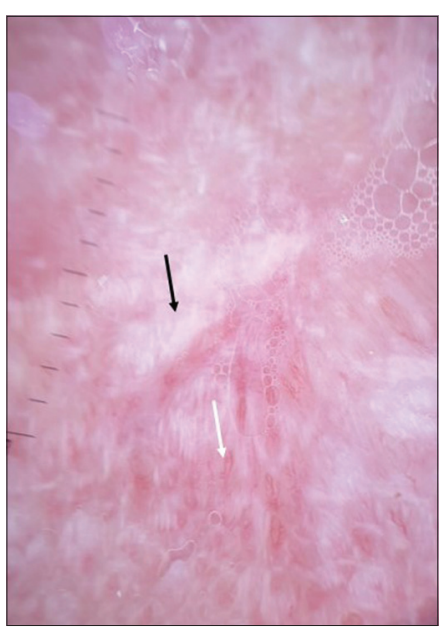

Figure 2: White patch with thin reticular striae (black arrow) surrounded with erythematous area and curved vessels (with arrow).

\section{DISCUSSION}

Lichen planus is a relatively common mucocutaneous disorder where cutaneous and mucosal lesions aren't always associated. Oral lichen planus (OLP) develops

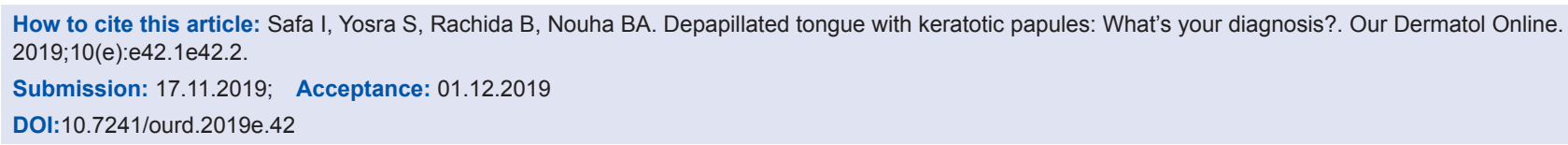




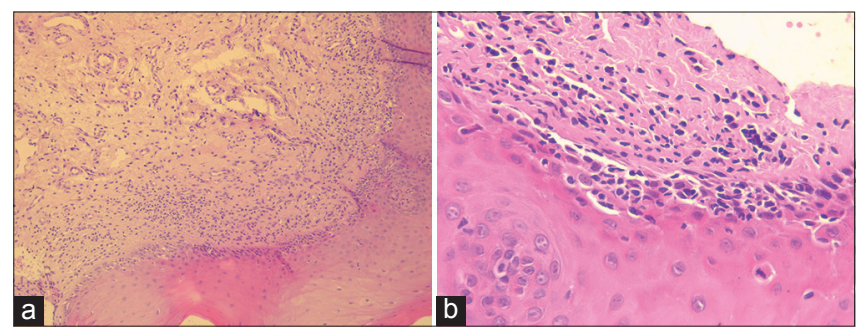

Figure 3: (a) Irregular acanthosis, basal layer vacuolization and necrotic keratinocytes $\left(\mathrm{HE}^{\star} 100\right)$. (b) moderately dense interface dermatitis (HE * 400).

in women more than twice as often as in men, with a mean age of onset in the sixth decade of life [1]. Buccal mucosa and tongue are most commonly affected, followed by gums and labial mucosa [2]. OLP isolated to a single oral site other than the gingiva is unusual, although occasional cases of isolated lesions on the tongue or on the lips have been reported [3].

Recent classifications group OLP lesions into reticular (reticular, papular, plaque-like), erythematous (atrophic), and erosive (ulcerative, bullous) forms [4].

To the best of our knowledge, mucoscopy of lingual lichen has been described in one report revealing a tri-colored pattern with structureless veil-like grey-white areas corresponding to modified Wickham's striae [5]. Like lichen planus, these structures display complete specificity for the correspondent dermatosis [6]. Other structures were reported to be found in OLP such as well-demarcated red glossy erosions, and violaceous-to-brown clods. The vascular pattern consists of dotted, linear and curved vessels along the borders of leukoplakia-like areas [6].

The therapy mainly aims at improvement of symptoms and regular observation of dysplastic changes. Topical glucocorticoids are the first-line treatement usually for erosive OLP, although systemic and intralesional steroids are also in use [7].

Our patient was prescribed topical application of low-potency steroid with great response.

\section{REFERENCES}

1. Eisen D. The clinical manifestations and treatment of oral lichen planus. Dermatologic Clinics. 2003;21:79-89.

2. Silverman SJr, Gorsky M, Lozada-Nur F. A prospective follow-up study of 570 patients with oral lichen planus: Persistence, remission, and malignant association. Oral Surg Oral Med Oral Pathol. 1985;60:3034.

3. Andreason JO. OLP: A clinical evaluation of 115 cases. Oral Surg Oral Med Oral Pathol. 1968;25:31-41.

4. Eisen D. The clinical manifestations and treatment of OLP. Dermatol Clinic. 2003;21:79-89.

5. Sonthalia S, Varma S, Jha AK, Jakhar D, Kaliyadan F. Case Report: Dermoscopic features of oral lichen planus - the evolution of mucoscopy. F1000Res. 2018;7:284.

6. Litaiem N, Mansour Y, Jones M, Zeglaoui F. Dermoscopic signs of lichen planus. BMJ Case Rep. 2016;2016:pii: bcr2015213923

7. Hasan S. Lichen planus of lip - Report of a rare case with review of literature. J Family Med Prim Care. 2019;8:1269-75.

Copyright by Idoudi Safa, et al. This is an open access article distributed under the terms of the Creative Commons Attribution License, which permits unrestricted use, distribution, and reproduction in any medium, provided the original author and source are credited.

Source of Support: Nil, Conflict of Interest: None declared. 